\title{
Molecular Aspects of Breast Cancer Metastasis to the Brain
}

\author{
Jodi M. Saunus, ${ }^{1}$ Majid Momeny, ${ }^{1}$ Peter T. Simpson, ${ }^{1}$ \\ Sunil R. Lakhani, ${ }^{1,2,3}$ and Leonard Da Silva ${ }^{1,2}$ \\ ${ }^{1}$ UQ Centre for Clinical Research, The University of Queensland, Herston, QLD 4029, Australia \\ ${ }^{2}$ School of Medicine, The University of Queensland, Herston, QLD 4029, Australia \\ ${ }^{3}$ Pathology Queensland, The Royal Brisbane and Women's Hospital, Herston, QLD 4029, Australia \\ Correspondence should be addressed to Leonard Da Silva, leonard@szd.com.br \\ Received 30 April 2011; Revised 15 July 2011; Accepted 14 August 2011 \\ Academic Editor: Sergio Roa
}

Copyright (๑) 2011 Jodi M. Saunus et al. This is an open access article distributed under the Creative Commons Attribution License, which permits unrestricted use, distribution, and reproduction in any medium, provided the original work is properly cited.

\begin{abstract}
Our knowledge of the biology underlying the development of brain metastases (BM) from breast cancer has improved over the last decade due to large clinical epidemiological studies, animal models of metastasis, and the use of high-resolution gene expression profiling technologies. However, there are still major gaps in our understanding of the mechanisms utilized by breast cancer cells to colonize the brain microenvironment, thus our arsenal of therapies remains relatively nonspecific, and the prognosis for breast cancer patients with BM remains poor. Additional insights into these mechanisms are necessary to facilitate the development of new preventive and curative therapeutic regimens to block this fatal disease. This paper aims to provide a general overview for the readers of what has been achieved in this field of research and its translation into clinical practice to date and to highlight exciting new areas of research that promise to inform the development of new targeted therapies for BM.
\end{abstract}

\section{An Overview of Metastasis}

Metastasis, or metastatic disease, is the spread of cancer cells from one organ to a distant site via the blood or lymph. In the Nineteenth Century, Paget asked whether the distribution of metastases in different organs was simply a matter of chance. He studied autopsy records of women with breast cancer, revealing a nonrandom pattern of metastatic colonization. $\mathrm{He}$ proposed a hypothesis that tumour cells (the "seed") could have specific affinity for the microenvironments of certain organs (the "soil") [1]. This intriguing phenomenon is called organotropism, and Paget's hypothesis has now been repeatedly substantiated, with growing recognition of the importance of tumour cell interactions with the stromal microenvironment in supporting the establishment of metastases (see below). For instance, analysis of large autopsy series has showed that lung, breast, melanoma, renal, and colon cancers are the most common primary tumours to metastasize to the brain $[2,3]$. There is a theory that the primary tumour could contribute to priming "premetastatic niches" prior to the establishment of micromestastases, thereby influencing organ tropism. Kaplan, Lyden, and colleagues' analysis of mouse models of lung metastasis implicated certain chemokines in this process, as well as mobilisation of haematopoietic precursor cells to pre-metastatic sites [4], however the mechanisms underlying creation of pre-metastatic niches are not fully understood [5].

The metastatic process is very inefficient. In order to accomplish distant metastasis, tumour cells must first detach and/or escape from the primary site, then survive as circulating tumour cells (CTCs) in the absence of the microenvironment cues with which they were conceived. Most CTCs are cleared from initial trapping sites within a few days. Those that survive, and succeed in extravasating, engraft at a distant site forming a micrometastasis, then may proliferate to form a clinically significant lesion after a fairly unpredictable period of latency (dormancy) spent meeting requirements for cell division in the new microenvironment $[6,7]$. The poorly understood "dormancy" phenomenon poses a major challenge in metastasis management. Despite being attractive drug targets (at least conceptually), CTCs and micrometastases are undetectable with current hematologic and imaging technologies and are thought to be insensitive to chemotherapeutics that target rapidly dividing cells. 
It is now becoming clear that only a very small proportion of primary tumour cells are capable of forming clinically significant metastatic lesion [8]. Because these cells share critical features with normal stem cells (namely, multi-lineage potential in reseeding a secondary tumour and selfrenewal), they have been called cancer stem cells (CSCs). It is hypothesized that a complex combination of tumour cell intrinsic and extrinsic processes eventually culminates in the activation of dormant CSC proliferation at distant sites [8]. Current evidence suggests that this includes continuing tumour cell genetic evolution, adaptation, and selection of those cells with signaling programs that best engage and exploit the new microenvironment $[9,10]$. Observations on the temporal courses of metastasis, from different primary tumour types, provide some insight into the biology underlying these processes. For example, subsets of breast and lung carcinomas show similar overall organ tropism (brain, bone, lung, and liver), but strikingly different progression times, with distant relapse detected early in lung cancer (macrometastases established within months of diagnosis), and relatively late in breast cancer progression (after years to decades of remission). This suggests early acquisition of metastasis-enabling genetic alterations in lung cancer, in contrast to a longer latency period as CTCs and/or micrometastases in breast cancer.

At least three categories of metastasis genes have been proposed to facilitate the multistep metastatic cascade (reviewed in [11]): (1) "initiation" genes that facilitate detachment (e.g., CDH2 (encodes N-Cadherin) and TWIST), extracellular matrix degradation (e.g., $M M P$ s) or angiogenesis (e.g., VEGF); (2) "progression" genes (e.g., PTGS2 (encodes COX-2) and MMP-1) that regulate extravasation of circulating tumour cells and are involved in metastatic colonisation; (3) "virulence" genes (e.g., IL6 and TNF $\alpha$ ), which promote survival in circulation, and/or provide a proliferative advantage in the distant microenvironment. Apart from these metastasis-promoting genes, there is a well-distinguished class of metastasis "suppressor" genes that represses tumour cell dissemination without any effect on primary tumour growth, including KAI-1, BRSM1, and NME1 [11]. These findings are mainly derived from studies using animal models. Historically, it has been difficult to understand the genetic alterations underlying metastasis by direct analysis of human tissue. Transcriptomic profiling of matched pairs of primary tumours and their metastases has demonstrated a high degree of similarity with minor differential gene expression [12-14]. However, the more recent application of next-generation whole genome sequencing (WGS) technologies is revealing subtle, but significant distinctions between metastases and their primary tumours of origin $[15,16]$ that were previously undetectable.

\section{What Is the Impact of Brain Metastasis in the Natural History of Breast Cancer?}

The prevalence of $\mathrm{BM}$ during the course of breast cancer disease has been reported to range from $10 \%-16 \%$, reaching $30 \%$ when autopsy diagnoses of $\mathrm{BM}$ are included $[17,18]$. Several factors have been reported to be associated with a higher risk of developing BM: patients less than fifty years old, four or more axillary lymph nodes involved with metastatic disease, basal phenotype, and high tumour grade [19-22]. Current therapeutic strategies for BM include whole brain radiation therapy (WBRT; the treatment mainstay since the 1950s), stereotactic radiosurgery, or surgery combined with radiotherapy. The median survival in untreated breast cancer patients with symptomatic BM is less than one month, $6-8$ weeks in patients treated with steroids alone and 3-6 months when treated with WBRT $[3,23]$.

Patients with HER2-positive breast tumours are now also regarded to be at high risk for developing BM. Improved control of systemic disease with the anti-HER2 monoclonal antibody combined with poor BBB penetration have been regarded as culprits of this increased metastatic trend [24]. BM treatment strategies are currently informed by the histopathology of the primary tumour, and BM are rarely biopsied. Whilst many features are shared, it is becoming clear that metastases are distinct in their genetic landscape and expression of critical disease markers [12, 14-16]. Hence, future therapeutic development is likely to be based on features of the metastases themselves.

\section{Does the Incidence of BM Correlate with Breast Cancer Molecular Subtypes?}

Breast cancer is a heterogeneous disease with respect to molecular features perhaps best exemplified by the molecular subgroups identified by gene expression profiling including basal-like, luminal A (hormone receptor positive), luminal B, and HER2 amplified/over-expressed (HER2+) subtypes. These molecular subtypes are associated with different outcomes. It is generally accepted that the basal-like, luminal B and HER2+ subtypes are associated with particularly poor prognosis compared with the hormone receptor-positive, luminal A subtype [25, 26] (Figure 1). Basal-like tumours are generally high grade, have central areas of necrosis, invasive pushing borders and are characterized by the expression of markers including high-molecular weight cytokeratins (e.g., CK14 and CK5/6), p53 and the myoepithelial markers Smooth Muscle Actin (SMA) and p63 [27, 28].

We, and other groups, have reported a greater propensity of primary breast cancer with a basal-like immunophenotype to metastasize to the brain [20-22]. Moreover, patients with germline $B R C A 1$ mutations who develop breast cancer have a higher incidence of $\mathrm{BM}$ compared to germline $B R C A 2$ carriers and non-BRCA1/2 patients [29]. These tumours have morphological similarities with those from the sporadic basal-like group [27, 28]. Patients with HER2+ tumours have also been shown to have an increased incidence of BM [30]. These metastatic patterns were further validated by a large study analysing, 3,726 cases, with a median follow-up of 14.8 years [31]. This work confirmed a higher rate of BM in HER2+-enriched and basal-like, compared to luminal A tumours. The cumulative incidence for BM in basal-like and HER2+ tumours were highest in the first five years after diagnosis, plateauing thereafter. 


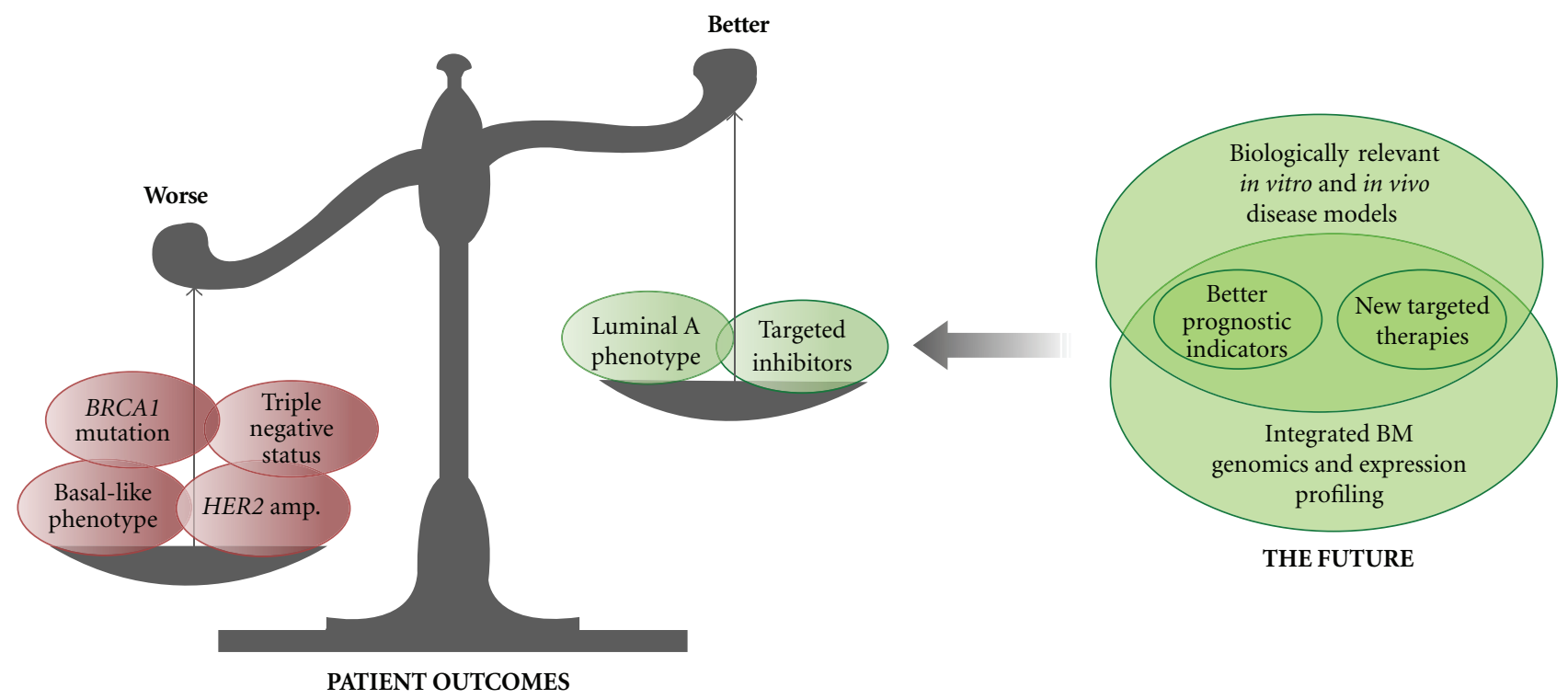

FIGURE 1: Summary of the major factors that increase or decrease risk of developing brain metastases in breast cancer and exciting new areas of research that promise to deliver the knowledge required for new targeted therapies and better prognostication.

\section{Current Understanding of $B M$ from Breast Cancer Mechanisms}

Gene expression profiling of breast tumours coupled with outcome data, functional analyses on cell lines, and in vivo animal models have shed light on our understanding of the colonization of the brain parenchyma by breast cancer cells [10, 11, 16, 42-44] (Figure 1).

Regarding the brain microenvironment, there are two main cell types in the neural tissue: neurons and glial cells, including microglia, astroglia, and oligodendroglia. There is a body of evidence suggesting that metastatic tumour cell interactions with the brain microenvironment facilitate the colonization process [43]. Interactions between breast cancer cells and pericytes and/or astrocytes might be responsible for alterations in the BBB and thus, development of BM from breast cancer. For example, Mendes et al. [45] reported that astrocyte-induced factors activated the ERK1/2 signalling pathway in rat mammary adenocarcinoma ENU1564 cells and thus enhanced the invasive features of these cells through increased expression of MMP-2. Furthermore, transfection of ENU1564 cells with TIMP-2, a natural inhibitor of MMP2 , reduced the in vitro invasive characteristics of these cells. BM was not observed in animals inoculated with ENU1564TIMP-2, which implies a cardinal role for MMP-2 in BMBC [45]. In addition, reactive glia under coculture with breast cancer cell line MDA-MB-231 was shown to enhance growth of this same cell line [46]. Furthermore, the 435-Br1 cell line, which was derived from BM in a nude mouse, showed increased adhesion to astrocytes and enhanced growth in vitro in the presence of an astrocyte-conditioned media when compared to parental MDA-MB-435 breast cancer cells and the lung metastasis-derived variant 435-Lung2 [47]. Table 1 summarizes BM-associated genes identified in integrated studies that used combinations of in vitro cell culture-based functional assays, in vivo mouse xenograft models and the analysis of human clinical samples.

Palmieri et al. demonstrated that when human MDAMB-231-BR cells, the brain metastatic derivative of the MDA-MB-231 cell line, were transfected with HER2, the HER2-overexpressing clones showed a threefold increase in the number of large BM compared with control MDA-MB231-BR cells [39]. These findings are consistent with epidemiological studies showing increased incidence of BM in HER2+ breast cancer patients [30]. The same group showed in vitro that Lapatinib inhibited the phosphorylation of EGFR, HER2, and downstream signalling proteins leading to reduced proliferation and migration in 231-BR. This in vitro data was also replicated in a mouse model showing Lapatinib inhibited the growth of brain macrometastases seeded from EGFR-overexpressing 231-BR cells [48].

Another example of an integrated approach to understanding BM from breast cancer was published by Bos et al. In this study, cyclooxygenase, the epidermal growth factor receptor ligand HBEGF and $\alpha 2,6$-sialyltransferase acted as mediators of cancer cell passage through the BBB. Again, these findings were derived from in vitro functional analyses and animal models, with translation in clinical samples where meta-analysis of gene expression profiling of patients who had brain metastases and survival data were combined. The presence of this signature was related to shorter brainmetastasis free survival [34].

Several studies focusing on the functional significance of single genes have also contributed to our current understanding of BM development. In an effort to develop an experimental model of BM from breast cancer, Kim et al. used internal carotid artery injection of breast cancer cells into nude mice, which resulted in formation of different brain metastatic variant cell lines. These variants expressed higher levels of vascular endothelial growth factor (VEGF) 
TABLE 1: Genes implicated in the development of brain metastases from breast cancer.

\begin{tabular}{|c|c|c|c|c|}
\hline Gene abbrev & Gene name & $\begin{array}{l}\text { BM expression } \\
\text { status }\end{array}$ & Gene product functions & Reference \\
\hline KCNMA1 & $\begin{array}{l}\text { Potassium large conductance calcium-activated } \\
\text { channel, subfamily } \mathrm{M} \text {, alpha member } 1\end{array}$ & $\uparrow$ & $\begin{array}{l}\text { Voltage gated ion channel involved } \\
\text { in neuronal excitability }\end{array}$ & {$[32]$} \\
\hline$M N T$ & MAX binding protein & $\downarrow$ & Myc antagonist & {$[33]$} \\
\hline TERC & Telomerase RNA component & $\uparrow$ & A template for telomere repeat & {$[33]$} \\
\hline CTSB & Cathepsin B & $\uparrow$ & Lysosomal cysteine proteinase & {$[33]$} \\
\hline PTGS2 & Prostaglandin-endoperoxide synthase 2 & $\uparrow$ & Prostaglandin biosynthesis & {$[34]$} \\
\hline HBEGF & Heparin-binding EGF-like growth factor & $\uparrow$ & EGFR signalling & {$[34]$} \\
\hline ST6GALNAC5 & $\begin{array}{l}\text { ST6 N-acetylgalactosaminide } \\
\text { alpha-2,6-sialyltransferase } 5\end{array}$ & $\uparrow$ & $\begin{array}{l}\text { Sialyltransferase that modifies } \\
\text { proteins and ceramides }\end{array}$ & {$[34]$} \\
\hline CXCR4 & Chemokine (C-X-C motif) receptor 4 & $\uparrow$ & $\begin{array}{l}\text { Receptor for stromal cell-derived } \\
\text { factor-1 }\end{array}$ & {$[35]$} \\
\hline MMP2 & Matrix metallopeptidase 2 & $\uparrow$ & Degradation of extracellular matrix & {$[36]$} \\
\hline MMP9 & Matrix metallopeptidase 9 & $\uparrow$ & Degradation of extracellular matrix & {$[36]$} \\
\hline ROBO1 & Roundabout & $\uparrow$ & $\begin{array}{l}\text { Axon guidance and neuronal } \\
\text { precursor cell migration }\end{array}$ & {$[37]$} \\
\hline$E R B B 3$ & $\begin{array}{l}\text { Y-erb-b2 erythroblastic leukemia viral } \\
\text { oncogene homolog } 3\end{array}$ & $\uparrow$ & $\begin{array}{l}\text { Cell proliferation and } \\
\text { differentiation }\end{array}$ & {$[12]$} \\
\hline CAV1 & Caveolin 1, caveolae protein & $\downarrow$ & $\begin{array}{l}\text { Structural component of the } \\
\text { caveolae plasma membranes }\end{array}$ & {$[38]$} \\
\hline$E R B B 2$ & $\begin{array}{l}\text { v-erb b2 erythroblastic leukemia viral } \\
\text { oncogene homolog } 2\end{array}$ & $\uparrow$ & Cell proliferation & [39] \\
\hline HK2 & Hexokinase 2 & $\uparrow$ & Glycolysis & {$[34]$} \\
\hline HPSE & Heparanase & $\uparrow$ & $\begin{array}{l}\text { Remodeling of the extracellular } \\
\text { matrix }\end{array}$ & {$[40]$} \\
\hline STAT3 & $\begin{array}{l}\text { Signal transducer and activator of } \\
\text { transcription } 3\end{array}$ & $\uparrow$ & $\begin{array}{l}\text { Cell growth and apoptosis, } \\
\text { inflammation, invasion, and } \\
\text { metastasis }\end{array}$ & {$[39]$} \\
\hline VEGF & Vascular endothelial growth factor & $\uparrow$ & $\begin{array}{l}\text { Stimulates angiogenesis and } \\
\text { vasculogenesis }\end{array}$ & {$[41]$} \\
\hline IL-8 & Interleukin 8 & $\uparrow$ & $\begin{array}{l}\text { CXC chemokine involved in } \\
\text { neutrophil recruitment }\end{array}$ & {$[41]$} \\
\hline
\end{tabular}

$\uparrow$ overexpressed; $\downarrow$ downregulated.

and IL-8 than the nonbrain metastatic clones, suggesting a possible role for VEGF in BM from breast cancer [40]. Consistent with this, suppression of $\mathrm{BM}$ and induction of apoptosis were observed following treatment of the mice with the VEGF-receptor tyrosine kinase inhibitor, PTK787/Z 222584 [40]. A second study by Palmieri et al. analyzed gene expression profiles of laser-captured epithelial cells from BM of breast cancer patients compared with unmatched primary breast tumors. The results showed that hexokinase 2 (HK2), a critical enzyme in glucose metabolism, is increased in $\mathrm{BM}$ [49]. Increased expression of HK2 was observed in the 231-BR brain metastastic breast cancer cell line [49]. A third example is the study by Chiu et al., which showed augmented expression of the activated form of signal transducer and activator of transcription 3 (STAT3) coupled with downregulation of Caveolin-1 (the structural component of caveolae involved in membrane trafficking and cell signaling) in BM compared with primary breast tumours. Furthermore, they showed that ectopic expression of Caveolin-1 or knockdown of STAT3 reduces the invasive features of breast cancer cells in vitro and brain colonization in vivo [38].
Our group has combined gene expression array profiling, targeted somatic mutation analysis, and immunohistochemical profiling of BM from breast cancer and integrated the data to demonstrate activation of signaling pathways associated with the HER receptor family in BM compared to their matched primary breast tumours. Critically, the data showed an increase in HER3 expression in breast cancer cells isolated from BM compared to matched primary tumours [12]. Neuregulin 1 , the ligand for this receptor, is abundantly expressed in the brain [50] and is activated by a variety of stimuli, including hypoxia [51]. Consistent with this, we observed increased expression of hypoxia-inducible Factor $1 \alpha$ (HIF$1 \alpha$ ) in the BM, likely reflecting the local hypoxic environment. Increased HER3 expression has also been reported in BM from lung cancer [52]. These two clinical snapshots could reflect environmental selection and therefore adaptation of metastatic cells to the brain microenvironment, exploiting the Neuregulin-HER3 axis in order to succeed.

While there is ample evidence that miRNAs have determinant roles in tumour cell dissemination, the possible roles of these "micromanagers of metastasis" in BM from breast 
cancer are poorly understood. Using miRanda for target prediction, Zhang et al. showed that miRNA-1258 targets heparanase (HPSE) which is the dominant endoglycosidase in mammals. HPSE favors tumor cell spread through dissolution of the extracellular matrix. In addition, this study introduced an inverse association between the miR-1258 levels and heparanase expression and enzymatic activity [53]. These findings were confirmed when the authors compared miRNA-1258 levels in clinical samples of invasive ductal carcinoma and BMBC compared with the corresponding normal or primary tissues. Moreover, the expression of miR1258 in BM from breast cancer cells has been shown to suppress heparanase in vitro cell invasion and experimental BM.

\section{The Blood-Brain-Barrier Is an Additional Challenge for BM Therapeutic Development}

The BBB comprises a specialized endothelium surrounded by a thick basement membrane and astrocytic endfeet [48]. Compared with endothelial cells from other vascular beds, brain microvascular endothelial cells characteristically have very low permeability to solutes and hydrophilic molecules, high electrical resistance, complex tight junctions, and an array of metabolic and transport systems that supply the brain with nutrients and eliminate brain metabolic by-products. The low permeability is important in protecting the brain from circulating toxins and restricting the migration of leukocytes and monocytes, consequently it is also hindrance to drug access. The BBB endothelia specifically express efflux transporters that further diminish the brain availability of certain chemotherapeutic agents, including p-glycoprotein, breast cancer resistance p-glycoprotein, and multidrug resistance-associated protein [54]. Moreover, binding of specific plasma proteins to chemotherapeutic drugs has been shown to decrease the concentrations of such drugs and subsequently, delivery of these drugs to the brain [41]. Therefore, poor drug delivery across the BBB is thought to be a major factor underlying the limited efficiency of systemic chemotherapies against BM from breast cancer, particularly monoclonal antibodies like Trastuzumab [48]. Large hydrophilic molecules, such as chemotherapeutic and molecular-targeted drugs, are excluded from the central nervous system unless they can be actively transported by receptor-mediated transcytosis. This highlights the need for new brain-permeable drugs.

In order to metastasize to the brain, breast cancer cells must attach to and invade through the BBB. A widely supported hypothesis is that breast tumour cell adhesion induces retraction of the endothelium, which exposes the vascular basement membrane to the breast cancer cells. There is ample evidence that breast cancer cells recognize and bind to components in the vascular membrane, thereby initiating extravasation and the beginning of colonization at secondary organ sites [43]. The impairment of the BBB was observed recently in breast cancer patients who developed metastasis to the brain. CXCR4/SDF-1 $\alpha$ has been suggested to play a major role in penetration of breast cancer cells through brain microvascular endothelial cells. In this regard, it has been reported that SDF-1- $\alpha$-mediated blood vessel instability via enhanced vascular permeability facilitates BM from breast cancer in a PI3K/Akt dependent manner [35].

The characteristics of the BBB necessitate the use of specialized, biologically relevant (and preferably throughput) preclinical models to test the efficacy of therapeutics against BM. Neural-cancer cell co-cultures can be used to simulate a brain-like microenvironment in vitro [55], but these simple models do not test whether compounds could cross the BBB in vivo. Bos and colleagues have developed a more biologically relevant in vitro transmigration assay, in which cancer cells adhere to and migrate across a human umbilical vein endothelial cell barrier towards human astrocytes segregated in a transwell chamber [34]. The gold standard preclinical model would involve xenograft transplantation of human $\mathrm{BM}$ into immunocompromised mice, but development is limited by the availability of fresh human tissue, and to our knowledge, none have been developed using metastatic cells selected in the human brain microenvironment. Alternative models have been generated by in vivo selection of neurotropic clones in nude mice, following intracardiac injection of metastatic cells from breast (231-BR, MCF7-HER2-BR, CN34-BM, and the mouse mammary tumour-derived 4T1 syngeneic model) [56, 57]. Table 1 summarizes a series of genes derived from integrated studies that have been implicated in BM development.

\section{Treatment Strategies against Metastatic Breast Cancers Including Brain Metastases}

We are approaching an era of personalized medicine, where therapies will be routinely tailored to individual tumours based on molecular diagnostics. Several targeted therapies for metastatic breast cancer are currently used or in clinical trials (Table 2; examples discussed below).

A recent phase II clinical trial using Lapatinib (dual inhibitor of HER1/2 with good brain penetration) in HER2+ breast cancer patients with BM despite prior radiation and Trastuzumab therapy showed Lapatinib has modest CNS antitumour activity [68]. This was corroborated in a mouse model where Lapatinib inhibited the colonisation of 231BR cells overexpressing EGFR and HER2 [48]. There is also evidence of synergism with Capecitabine, a pyrimidine analogue prodrug approved for metastatic breast cancer management $[41,54]$. Sunitinib, a multikinase inhibitor (targets include VEGF-Rs, c-kit and PDGF-Rs), is also under investigation for its effects on BM in metastatic breast and renal cell carcinomas $[64,65,69]$.

\section{Possible "Druggable" Targets for the Future in BM from Breast Cancer}

A recent study has demonstrated the role of STAT3 in BM from breast cancer [38]. Moreover, it has been shown that STAT3 inhibition by restoration of its inhibitor, suppressor of cytokine signalling (SOCS-1), results in induction of Caveolin-1, a tumour suppressor gene in breast cancer [38]. In this regard, STAT3 might be an attractive therapeutic target in BM from breast cancer. 
TABLE 2: Current targeted therapies and their use in metastatic breast cancer (MBC).

\begin{tabular}{|c|c|c|c|c|}
\hline Drug & Class & Targets & Clinical indications & Reference \\
\hline Trastuzumab & $\mathrm{mAb}$ & HER2 & $\begin{array}{l}\text { Single agent for HER2+ MBC; used in combination } \\
\text { with paclitaxel as first-line therapy for HER2+ MBC }\end{array}$ & {$[58]$} \\
\hline Lapatinib & TKI & HER1/2 & $\begin{array}{l}\text { Active in Trastuzumab-resistant, HER } 2+\text { breast } \\
\text { cancer; crosses the BBB and suppresses CNS } \\
\text { metastasis used in combination with Capecitabine } \\
\text { for HER } 2+\text { MBC }\end{array}$ & {$[41]$} \\
\hline Pertuzumab & $\mathrm{mAb}$ & HER2 & $\begin{array}{l}\text { Impairs HER2 homo-/hetero-dimerisation; active in } \\
\text { Trastuzumab-resistant HER2+ breast cancers; not } \\
\text { currently approved for MBC }\end{array}$ & {$[59]$} \\
\hline Neratinib & TKI & HER1/2 & $\begin{array}{l}\text { Inhibits HER2 autophosphorylation and suppresses } \\
\text { downstream signalling; active in HER2+ patients } \\
\text { with and without Trastuzumab pretreatment; not } \\
\text { currently approved for MBC }\end{array}$ & {$[60]$} \\
\hline Bevacizumab & $\mathrm{mAb}$ & VEGF & $\begin{array}{l}\text { Antiangiogenic therapy for MBC in combination } \\
\text { with Docetaxel or Paclitaxel for first-line treatment }\end{array}$ & {$[61]$} \\
\hline Sorafenib & TKI & $\begin{array}{l}\text { VEGFR } \\
\text { PDGFR } \\
\text { Raf }\end{array}$ & $\begin{array}{l}\text { Multitarget receptor tyrosine kinase inhibitor; } \\
\text { FDA-approved for advanced renal cancer and } \\
\text { hepatocellular carcinoma }\end{array}$ & {$[62]$} \\
\hline Axitinib & TKI & $\begin{array}{l}\text { VEGFR } \\
\text { PDGFR }\end{array}$ & $\begin{array}{l}\text { Currently in phase III clinical trial for metastatic } \\
\text { renal cell carcinoma }\end{array}$ & {$[63]$} \\
\hline Sunitinib & TKI & $\begin{array}{l}\text { VEGFR } \\
\text { PDGFR } \\
\text { KIT }\end{array}$ & $\begin{array}{l}\text { Antiangiogenic therapy; FDA-approved for renal cell } \\
\text { carcinoma and Gleevec-resistant gastrointestinal } \\
\text { stromal tumours; effective as a single agent for } \\
\text { metastatic breast cancer (phase II study) }\end{array}$ & {$[64-66]$} \\
\hline Pazopanib & TKI & $\begin{array}{l}\text { VEGFR } \\
\text { PDGFR } \\
\text { KIT }\end{array}$ & $\begin{array}{l}\text { Anti-angiogenic therapy; FDA-approved for renal } \\
\text { cell carcinoma; combination with Lapatinib has } \\
\text { superior activity as the first-line treatment for MBC } \\
\text { (phase II study) }\end{array}$ & {$[67]$} \\
\hline
\end{tabular}

Abbreviations: BBB: blood-brain barrier; CNS: central nervous system; HER1: Epidermal Growth Factor Receptor (EGFR); mAb: monoclonal antibody; KIT: Mast/stem cell growth factor receptor gene; PDGFR: platelet-derived growth factor receptor; TKI: tyrosine kinase inhibitor; VEGFR: vascular endothelial growth factor receptor.

Secondly, a growing body of evidence suggests that heparanase, a downstream target of EGFR/HER2, might be involved in BM from breast cancer [53]. EGF induces nucleolar translocation of HPSE and induction of DNA topoisomerase I which is essential for BM from breast cancer and cell proliferation [53]. In this setting, heparanase has been suggested as a potential target that could be exploited therapeutically [53].

Palmieri et al. [49] reported that hexokinase 2 (HK2) is significantly increased in brain metastases compared to unmatched primary breast tumors. Hexokinases phosphorylate glucose to produce glucose-6-phosphate in the first step of glucose metabolism. It is thought that HK2 increases glycolysis in tumor cells and may therefore be an attractive therapeutic target [49].

We recently identified somatic activating mutations in genes associated with the AKT and MAPK signaling pathways, including PIK3CA, KRAS, HRAS, and NRAS, in BM from breast and other cancers [12]. This highlights the possibility of cancer cells resisting targeted treatment to molecules such as HER2 or EGFR by acquiring oncogenic mutations in downstream pathways. Therapeutic modalities targeting these downstream pathways are currently being investigated [59]. In addition, Grigoriadis et al. demonstrated the presence of CT-X antigens in a cohort of BM, including BM from breast cancer. These proteins are predominantly expressed in human germ cells and not somatic tissues, but are frequently activated in cancer. Nearly two-thirds of our BM cohort showed expression of MAGE-A and NY-ESO, two of the CT$\mathrm{X}$ antigens for which inhibitors are currently in clinical trials for lung cancer and melanoma [70]. Targeting these antigens could therefore also be an effective therapeutic strategy for BM.

\section{Future Directions: Brain Metastases in the Whole Genome Sequencing Era}

Direct, high-resolution genomic and transcriptomic analyses of BM may uncover new druggable features that can be targeted to specifically inhibit BM cells. The development and continual refinement of WGS technologies has dramatically increased the resolution with which we can analyse cancer genomes. We can now screen for new mutations, simultaneously and accurately assess their frequencies and correlate this with expression data across the entire genome. Rare mutations, that are present in only a small percentage of cells within the heterogeneous primary tumour mass, may be important in progression and virulence acquisition at a metastatic site. Although there are no large-scale BM genomics studies published to date, Ding and colleagues used WGS 
to interrogate the genomic profiles of a human $\mathrm{BM}$, its corresponding primary breast tumour, and a mouse xenograft derivative [44]. They showed that; (1) most of the genomic changes present in the primary tumour are propagated during the clinical course of the disease; (2) the frequency of some mutations was increased in the BM, suggesting enrichment with a subpopulation of cells selected from a more heterogeneous primary tumour; (3) two different populations of cells from the primary tumour, with distinct subsets of mutations, were selected during the metastatic process. The third finding is striking and is consistent with other data suggesting that metastases may be established from clusters of cells, rather than one founder cell [71] or may derive from regions of the tumour where there is clonal heterogeneity [16].

\section{Conclusions}

Thus far, we have only fragmented knowledge of the mechanisms underlying breast cancer cell colonisation of the brain microenvironment. Animal models and gene expression profiling have and will continue to provide insight, however it is anticipated that next-generation sequencing technology and integration of this data with expression profiling will enable us to generate a comprehensive map of the brain metastasis genomic landscape. To be able to translate this impending knowledge into clinical outcomes, we must continue to develop and refine high-throughput, biologically relevant preclinical models that accurately mimic the brain microenvironment and the BBB (summarized in Figure 1).

\section{Acknowledgments}

Peter T. Simpson is a recipient of a fellowship from the National Breast Cancer Foundation (NBCF), Australia. The brain metastasis data reported herein was obtained by Leonard Da Silva during his Ph.D. funded by the Ludwig Institute for Cancer Research. J. M. Saunus and M. Momeny contributed equally to the paper.

\section{References}

[1] S. Paget, "The distribution of secondary growths in cancer of the breast. 1889," Cancer and Metastasis Reviews, vol. 8, no. 2, pp. 98-101, 1989.

[2] Y. Tsukada, A. Fouad, J. W. Pickren, and W. W. Lane, "Central nervous system metastasis from breast carcinoma. Autopsy study," Cancer, vol. 52, no. 12, pp. 2349-2354, 1983.

[3] W. Boogerd, V. W. Vos, A. A. M. Hart, and G. Baris, "Brain metastases in breast cancer; natural history, prognostic factors and outcome," Journal of Neuro-Oncology, vol. 15, no. 2, pp. 165-174, 1993.

[4] R. N. Kaplan, R. D. Riba, S. Zacharoulis et al., "VEGFR1positive haematopoietic bone marrow progenitors initiate the pre-metastatic niche," Nature, vol. 438, no. 7069, pp. 820-827, 2005.

[5] M. R. Dawson, D. G. Duda, D. Fukumura, and R. K. Jain, "VEGFR1-activity-independent metastasis formation," $\mathrm{Na}$ ture, vol. 461, no. 7262, pp. E4-E5, 2009.
[6] K. J. Luzzi, I. C. MacDonald, E. E. Schmidt et al., "Multistep nature of metastatic inefficiency: dormancy of solitary cells after successful extravasation and limited survival of early micrometastases," American Journal of Pathology, vol. 153, no. 3, pp. 865-873, 1998.

[7] R. L. Vessella, K. Pantel, and S. Mohla, "Tumor cell dormancy: an NCI workshop report," Cancer Biology and Therapy, vol. 6, no. 9, pp. 1496-1504, 2007.

[8] F. Li, B. Tiede, J. Massagué, and Y. Kang, "Beyond tumorigenesis: cancer stem cells in metastasis," Cell Research, vol. 17, no. 1, pp. 3-14, 2007.

[9] A. L. Allana, S. A. Vantyghem, A. B. Tuck, and A. F. Chambers, "Tumor dormancy and cancer stem cells: implications for the biology and treatment of breast cancer metastasis," Breast Disease, vol. 26, no. 1, pp. 87-98, 2006.

[10] L. A. Mina and G. W. Sledge Jr., "Rethinking the metastatic cascade as a therapeutic target," Nature Reviews Clinical Onco$\log y$, vol. 8, no. 6, pp. 325-332, 2011.

[11] D. X. Nguyen, P. D. Bos, and J. Massagué, "Metastasis: from dissemination to organ-specific colonization," Nature Reviews Cancer, vol. 9, no. 4, pp. 274-284, 2009.

[12] L. Da Silva, P. T. Simpson, C. E. Smart et al., "HER3 and downstream pathways are involved in colonization of brain metastases from breast cancer," Breast Cancer Research, vol. 12, no. 4, article R46, 2010.

[13] B. Weigelt, A. M. Glas, L. F. A. Wessels, A. T. Witteveen, J. L. Peterse, and L. J. Van'T Veer, "Gene expression profiles of primary breast tumors maintained in distant metastases," Proceedings of the National Academy of Sciences of the United States of America, vol. 100, no. 26, pp. 15901-15905, 2003.

[14] M. Vecchi, S. Confalonieri, P. Nuciforo et al., "Breast cancer metastases are molecularly distinct from their primary tumors," Oncogene, vol. 27, no. 15, pp. 2148-2158, 2008.

[15] S. P. Shah, R. D. Morin, J. Khattra et al., "Mutational evolution in a lobular breast tumour profiled at single nucleotide resolution," Nature, vol. 461, no. 7265, pp. 809-813, 2009.

[16] S. Yachida, S. Jones, I. Bozic et al., "Distant metastasis occurs late during the genetic evolution of pancreatic cancer," Nature, vol. 467, no. 7319, pp. 1114-1117, 2010.

[17] Y. Urano, T. Fukushima, S. Kitamura, H. Mori, K. Baba, and S. Aizawa, "Statistical studies on metastasis of breast cancer and cancer metastasis to the breast," Gan No Rinsho, vol. Suppl, pp. 205-223, 1986.

[18] N. U. Lin, J. R. Bellon, and E. P. Winer, "CNS metastases in breast cancer," Journal of Clinical Oncology, vol. 22, no. 17, pp. 3608-3617, 2004.

[19] Y. L. Tham, K. Sexton, R. Kramer, S. Hilsenbeck, and R. Elledge, "Primary breast cancer phenotypes associated with propensity for central nervous system metastases," Cancer, vol. 107, no. 4, pp. 696-704, 2006.

[20] L. G. Fulford, J. S. Reis-Filho, K. Ryder et al., "Basal-like grade III invasive ductal carcinoma of the breast: patterns of metastasis and long-term survival," Breast Cancer Research, vol. 9, no. 1, article R4, 2007.

[21] J. Gaedcke, F. Traub, S. Milde et al., "Predominance of the basal type and HER-2/neu type in brain metastasis from breast cancer," Modern Pathology, vol. 20, no. 8, pp. 864-870, 2007.

[22] D. G. Hicks, S. M. Short, N. L. Prescott et al., "Breast cancers with brain metastases are more likely to be estrogen receptor negative, express the basal cytokeratin CK5/6, and overexpress HER2 or EGFR," American Journal of Surgical Pathology, vol. 30, no. 9, pp. 1097-1104, 2006.

[23] P. K. Sneed, D. A. Larson, and W. M. Wara, "Radiotherapy for cerebral metastases," Neurosurgery Clinics of North America, vol. 7, no. 3, pp. 505-515, 1996. 
[24] Y. H. Park, M. J. Park, S. H. Ji et al., "Trastuzumab treatment improves brain metastasis outcomes through control and durable prolongation of systemic extracranial disease in HER2overexpressing breast cancer patients," British Journal of Cancer, vol. 100, no. 6, pp. 894-900, 2009.

[25] C. M. Perou, T. Sørile, M. B. Eisen et al., "Molecular portraits of human breast tumours,” Nature, vol. 406, no. 6797, pp. 747$752,2000$.

[26] T. Sørlie, C. M. Perou, R. Tibshirani et al., "Gene expression patterns of breast carcinomas distinguish tumor subclasses with clinical implications," Proceedings of the National Academy of Sciences of the United States of America, vol. 98, no. 19, pp. 10869-10874, 2001.

[27] L. Da Silva, C. Clarke, and S. R. Lakhani, "Demystifying basallike breast carcinomas," Journal of Clinical Pathology, vol. 60, no. 12, pp. 1328-1332, 2007.

[28] E. A. Rakha, J. S. Reis-Filho, and I. O. Ellis, "Basal-like breast cancer: a critical review," Journal of Clinical Oncology, vol. 26, no. 15 , pp. 2568-2581, 2008.

[29] L. Albiges, F. André, C. Balleyguier, G. Gomez-Abuin, A. Chompret, and S. Delaloge, "Spectrum of breast cancer metastasis in BRCA1 mutation carriers: highly increased incidence of brain metastases," Annals of Oncology, vol. 16, no. 11, pp. 1846-1847, 2005.

[30] N. U. Lin and E. P. Winer, "Brain metastases: the HER2 paradigm," Clinical Cancer Research, vol. 13, no. 6, pp. 1648-1655, 2007.

[31] H. Kennecke, R. Yerushalmi, R. Woods et al., "Metastatic behavior of breast cancer subtypes," Journal of Clinical Oncology, vol. 28, no. 20, pp. 3271-3277, 2010.

[32] D. Khaitan, U. T. Sankpal, B. Weksler et al., "Role of KCNMA1 gene in breast cancer invasion and metastasis to brain," BMC Cancer, vol. 9, article 258, 2009.

[33] I. Nishizuka, T. Ishikawa, Y. Hamaguchi et al., "Analysis of gene expression involved in brain metastasis from breast cancer using cDNA microarray," Breast Cancer, vol. 9, no. 1, pp. 26$32,2002$.

[34] P. D. Bos, X. H. F. Zhang, C. Nadal et al., "Genes that mediate breast cancer metastasis to the brain," Nature, vol. 459, no. 7249, pp. 1005-1009, 2009.

[35] B. C. Lee, T. H. Lee, S. Avraham, and H. K. Avraham, "Involvement of the chemokine receptor CXCR4 and its ligand stromal cell-derived factor $1 \alpha$ in breast cancer cell migration through human brain microvascular endothelial cells," Molecular Cancer Research, vol. 2, no. 6, pp. 327-338, 2004.

[36] O. Mendes, H. T. Kim, and G. Stoica, "Expression of MMP2, MMP9 and MMP3 in breast cancer brain metastasis in a rat model," Clinical and Experimental Metastasis, vol. 22, no. 3, pp. 237-246, 2005.

[37] B. C. Schmid, G. A. Rezniczek, G. Fabjani, T. Yoneda, S. Leodolter, and R. Zeillinger, "The neuronal guidance cue Slit2 induces targeted migration and may play a role in brain metastasis of breast cancer cells," Breast Cancer Research and Treatment, vol. 106, no. 3, pp. 333-342, 2007.

[38] W.-T. Chiu, H.-T. Lee, F.-J. Huang et al., "Caveolin-1 upregulation mediates suppression of primary breast tumor growth and brain metastases by Stat 3 inhibition," Cancer Research, vol. 71, no. 14, pp. 4932-4943, 2011.

[39] D. Palmieri, J. L. Bronder, J. M. Herring et al., "Her-2 overexpression increases the metastatic outgrowth of breast cancer cells in the brain," Cancer Research, vol. 67, no. 9, pp. 41904198, 2007.

[40] L. S. Kim, S. Huang, W. Lu, D. C. Lev, and J. E. Price, "Vascular endothelial growth factor expression promotes the growth of breast cancer brain metastases in nude mice," Clinical and Experimental Metastasis, vol. 21, no. 2, pp. 107-118, 2004.

[41] C. E. Geyer, J. Forster, D. Lindquist et al., "Lapatinib plus capecitabine for HER2-positive advanced breast cancer," New England Journal of Medicine, vol. 355, no. 26, pp. 2733-2743, 2006.

[42] I. J. Fidler, "The role of the organ microenvironment in brain metastasis," Seminars in Cancer Biology, vol. 21, no. 2, pp. 107112,2011

[43] I. J. Fidler, K. Balasubramanian, Q. Lin, S. W. Kim, and S.J. Kim, "The brain microenvironment and cancer metastasis," Molecules and Cells, vol. 30, no. 2, pp. 93-98, 2010.

[44] L. Ding, M. J. Ellis, S. Li et al., "Genome remodelling in a basallike breast cancer metastasis and xenograft," Nature, vol. 464, no. 7291, pp. 999-1005, 2010.

[45] O. Mendes, H. T. Kim, G. Lungu, and G. Stoica, "MMP2 role in breast cancer brain metastasis development and its regulation by TIMP2 and ERK1/2," Clinical and Experimental Metastasis, vol. 24, no. 5, pp. 341-351, 2007.

[46] D. P. Fitzgerald, D. Palmieri, E. Hua et al., "Reactive glia are recruited by highly proliferative brain metastases of breast cancer and promote tumor cell colonization," Clinical and Experimental Metastasis, vol. 25, no. 7, pp. 799-810, 2008.

[47] A. Sierra, J. E. Price, M. García-Ramirez, O. Méndez, L. López, and A. Fabra, "Astrocyte-derived cytokines contribute to the metastatic brain specificity of breast cancer cells," Laboratory Investigation, vol. 77, no. 4, pp. 357-368, 1997.

[48] B. Gril, D. Palmieri, J. L. Bronder et al., "Effect of lapatinib on the outgrowth of metastatic breast cancer cells to the brain," Journal of the National Cancer Institute, vol. 100, no. 15, pp. 1092-1103, 2008.

[49] D. Palmieri, D. Fitzgerald, S. M. Shreeve et al., "Analyses of resected human brain metastases of breast cancer reveal the association between up-regulation of hexokinase 2 and poor prognosis," Molecular Cancer Research, vol. 7, no. 9, pp. 14381445, 2009.

[50] A. J. Law, C. Shannon Weickert, T. M. Hyde, J. E. Kleinman, and P. J. Harrison, "Neuregulin-1 (NRG-1) mRNA and protein in the adult human brain," Neuroscience, vol. 127, no. 1, pp. 125-136, 2004.

[51] G. L. Wang and G. L. Semenza, "General involvement of hypoxia-inducible factor 1 in transcriptional response to hypoxia," Proceedings of the National Academy of Sciences of the United States of America, vol. 90, no. 9, pp. 4304-4308, 1993.

[52] M. Sun, C. Behrens, L. Feng et al., "HER family receptor abnormalities in lung cancer brain metastases and corresponding primary tumors," Clinical Cancer Research, vol. 15, no. 15, pp. 4829-4837, 2009.

[53] L. Zhang, P. S. Sullivan, J. C. Goodman, P. H. Gunaratne, and D. Marchetti, "MicroRNA-1258 suppresses breast cancer brain metastasis by targeting heparanase," Cancer Research, vol. 71, no. 3, pp. 645-654, 2011.

[54] N. U. Lin, V. Diéras, D. Paul et al., "Multicenter phase II study of lapatinib in patients with brain metastases from HER2positive breast cancer," Clinical Cancer Research, vol. 15, no. 4, pp. 1452-1459, 2009.

[55] S.-J. Kim, J.-S. Kim, E. S. Park et al., "Astrocytes upregulate survival genes in tumor cells and induce protection from chemotherapy," Neoplasia, vol. 13, no. 3, pp. 286-298, 2011.

[56] S. Ostrand-Rosenberg, "Mouse SaI sarcoma tumor model," Current Protocols in Immunology, vol. Chapter 20, p. Unit 20.3, 2001.

[57] T. Yoneda, P. J. Williams, T. Hiraga, M. Niewolna, and R. Nishimura, "A bone-seeking clone exhibits different biological 
properties from the MDA-MB-231 parental human breast cancer cells and a brain-seeking clone in vivo and in vitro," Journal of Bone and Mineral Research, vol. 16, no. 8, pp. 14861495, 2001.

[58] H. J. Burstein, I. Kuter, S. M. Campos et al., "Clinical activity of trastuzumab and vinorelbine in women with HER2-overexpressing metastatic breast cancer," Journal of Clinical Oncology, vol. 19, no. 10, pp. 2722-2730, 2001.

[59] J. Baselga, K. A. Gelmon, S. Verma et al., "Phase II trial of pertuzumab and trastuzumab in patients with human epidermal growth factor receptor 2-positive metastatic breast cancer that progressed during prior trastuzumab therapy," Journal of Clinical Oncology, vol. 28, no. 7, pp. 1138-1144, 2010.

[60] H. J. Burstein, Y. Sun, L. Y. Dirix et al., "Neratinib, an irreversible ErbB receptor tyrosine kinase inhibitor, in patients with advanced ErbB2-positive breast cancer," Journal of Clinical Oncology, vol. 28, no. 8, pp. 1301-1307, 2010.

[61] K. Miller, M. Wang, J. Gralow et al., "Paclitaxel plus bevacizumab versus paclitaxel alone for metastatic breast cancer," New England Journal of Medicine, vol. 357, no. 26, pp. 26662676, 2007.

[62] B. Escudier, T. Eisen, W. M. Stadler et al., "Sorafenib in advanced clear-cell renal-cell carcinoma," New England Journal of Medicine, vol. 356, no. 2, pp. 125-134, 2007.

[63] T. K. Choueiri, "Axitinib, a novel anti-angiogenic drug with promising activity in various solid tumors," Current Opinion in Investigational Drugs, vol. 9, no. 6, pp. 658-671, 2008.

[64] R. Addeo and M. Caraglia, "The oral tyrosine kinase inhibitors lapatinib and sunitinib: new opportunities for the treatment of brain metastases from breast cancer?" Expert Review of Anticancer Therapy, vol. 11, no. 2, pp. 139-142, 2011.

[65] F. Petrelli, M. Cabiddu, M. Carpo, M. Ghilardi, and S. Barni, "Progression of intramedullary metastasis during perioperative cessation of sunitinib," Nature Reviews Urology, vol. 7, no. 11, pp. 634-637, 2010.

[66] R. J. Motzer, T. E. Hutson, P. Tomczak et al., "Sunitinib versus interferon alfa in metastatic renal-cell carcinoma," New England Journal of Medicine, vol. 356, no. 2, pp. 115-124, 2007.

[67] T. E. Hutson, I. D. Davis, J. P. H. Machiels et al., "Efficacy and safety of pazopanib in patients with metastatic renal cell carcinoma," Journal of Clinical Oncology, vol. 28, no. 3, pp. 475-480, 2010.

[68] N. U. Lin, L. A. Carey, M. C. Liu et al., "Phase II trial of lapatinib for brain metastases in patients with human epidermal growth factor receptor 2-positive breast cancer," Journal of Clinical Oncology, vol. 26, no. 12, pp. 1993-1999, 2008.

[69] U. M. Vogl, M. Bojic, W. Lamm et al., "Extracerebral metastases determine the outcome of patients with brain metastases from renal cell carcinoma," BMC Cancer, vol. 10, article 480, 2010.

[70] A. Grigoriadis, O. L. Caballero, K. S. Hoek et al., "CT-X antigen expression in human breast cancer," Proceedings of the National Academy of Sciences of the United States of America, vol. 106, no. 32, pp. 13493-13498, 2009.

[71] W. Lu, C. D. Bucana, and A. J. Schroit, "Pathogenesis and vascular integrity of breast cancer brain metastasis," International Journal of Cancer, vol. 120, no. 5, pp. 1023-1026, 2007. 

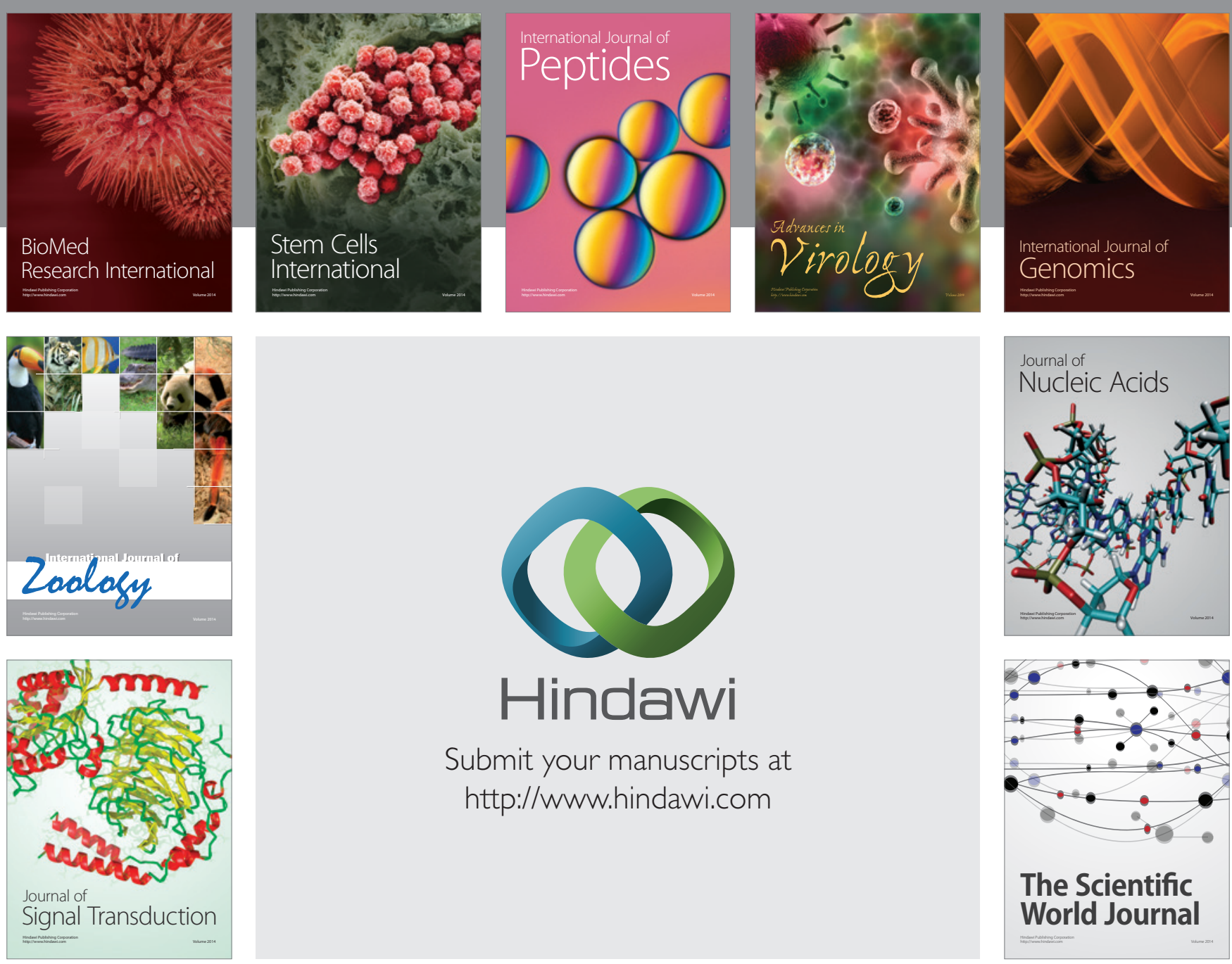

Submit your manuscripts at

http://www.hindawi.com
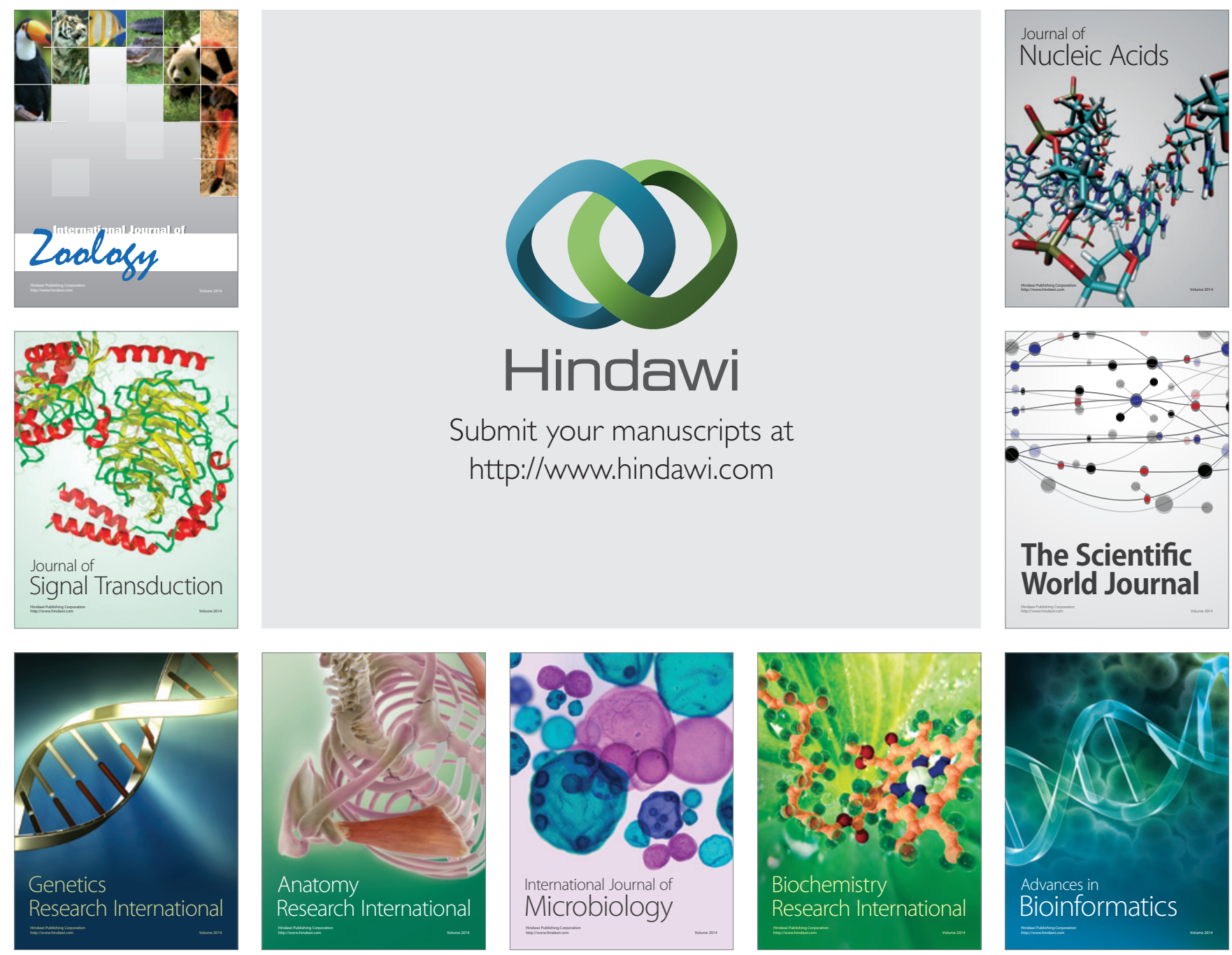

The Scientific World Journal
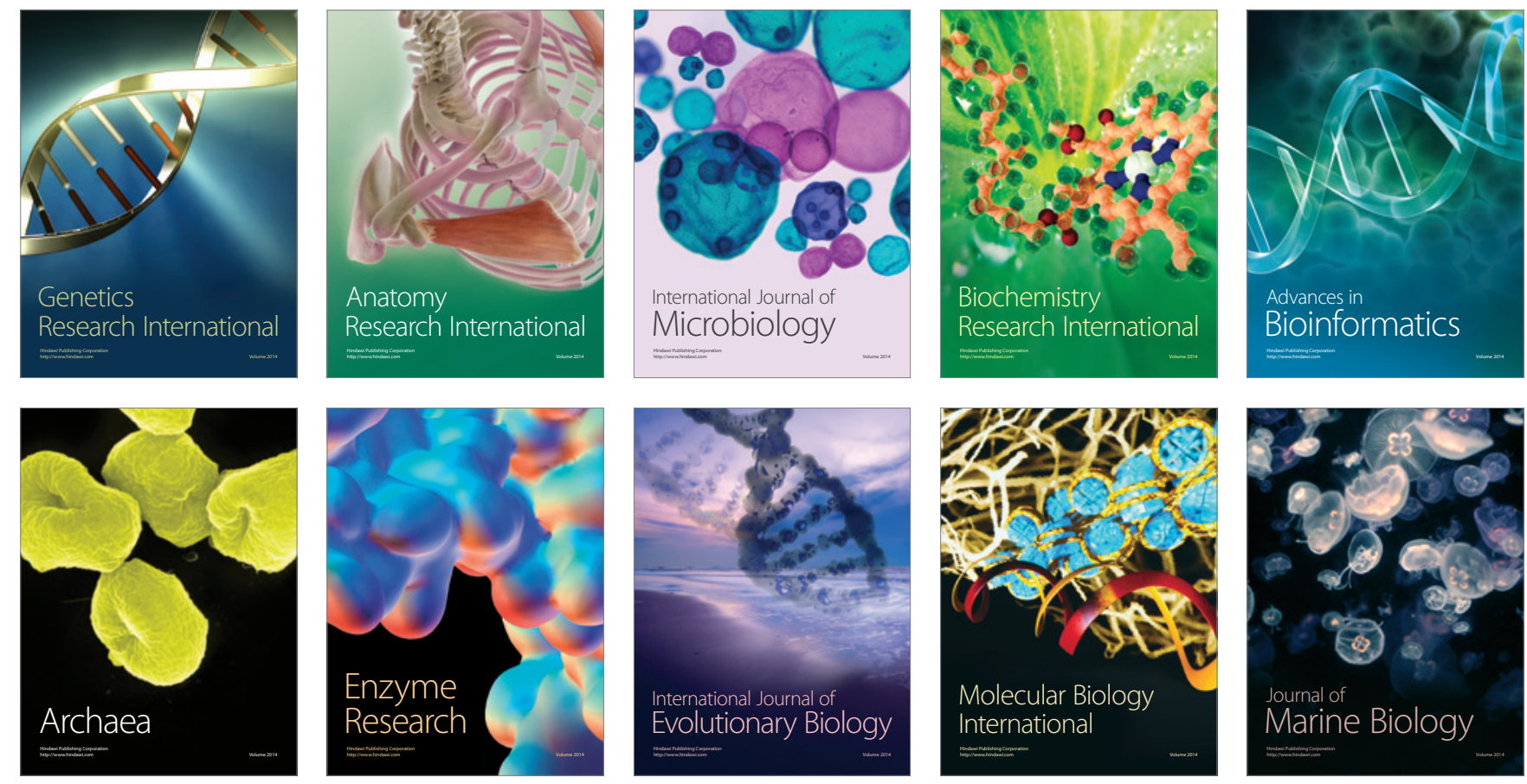